\title{
le frottement dans le renforcement des sols
}

\author{
par \\ F. Schlosser \\ Professeur de Mécanique des Sols à l'École Nationale des Ponts et Chaussées \\ Directeur de TERRASOL \\ A. Guilloux \\ Ingénieur à TERRASOL
}

\section{Introduction}

Le renforcement des sols regroupe un ensemble de techniques consistant à améliorer la résistance initiale du sol par l'inclusion d'éléments linéaires résistants. généralement métalliques. Le phénomène d'interaction entre le sol et l'inclusion est fréquemment le frottement et dans ce cas le renforcement n'est possible et économique que si le sol possède un bon squelette granulaire permettant de développer un frottement interne à court terme. La figure 1 donne pour les trois types de renforcement fonctionnant par frottement (terre armée, cloutage, micropieux) les efforts qui se développent dans l'inclusion.

\begin{tabular}{|c|c|c|c|}
\hline \multirow[b]{2}{*}{$\begin{array}{l}\text { Effort } \\
\text { dans } \\
\text { l'inclusion }\end{array}$} & \multicolumn{3}{|c|}{ Type de renforcement } \\
\hline & $\begin{array}{l}\text { Terre } \\
\text { Armée }\end{array}$ & Cloutage & Micropieux \\
\hline Traction & $\star$ & $\star$ & $\star$ \\
\hline Compression & & & $\star$ \\
\hline Flexion & & $\star$ & $\star$ \\
\hline
\end{tabular}

Si l'effort de flexion est dû aux contraintes normales $\sigma$ exercées par le sol sur la surface latérale de l'inclusion, les efforts de traction et de compression résultent par contre des contraintes de cisaillement $\tau$ sur cette surface. La figure 2 montre le mécanisme de fonctionnement des trois types de renforcement et les courbes caractéristiques de distribution des efforts de traction et de compression. Le dimensionnement des inclusions requiert la connaissance de la contrainte $\tau$, ou tout au moins de la valeur limite de cette contrainte, pour toute valeur de la contrainte normale $\sigma$, c'est-à-dire en fait la connaissance du frottement mobilisable entre le sol et l'inclusion.
On présente ici une étude de ce frottement en mettant l'accent sur les phénomènes physiques, sur les principaux facteurs en jeu, sur les méthodes de mesure, et sur les règles de dimensionnement pratiques.

\section{Aspects particuliers du frottement sol- inclusion}

Le frottement sol-inclusion dans un sol grenu diffère sensiblement du frottement classique plan sur plan sous effort normal constant. Sa particularité est due essentiellement à son caractère tridimensionnel, qui rend complexes à la fois son analyse et sa prévision. Par suite de cet aspect tridimensionnel il y a une réponse de la masse de sol, lors de la mobilisation du frottement, qui se traduit comme on le verra par une augmentation sensible de la contrainte normale $\sigma$ qui s'exerce sur l'inclusion. L'analyse ne peut plus dès lors se faire à partir de la loi élémentaire simple du frottement, puisqu'il n'y a plus unicité de la loi effort-déplacement.

L'inclusion elle aussi réagit par sa déformabilité relative qui influence la répartition des contraintes de cisaillement.

Le frottement sol-inclusion ne peut donc pas être étudié comme un phénomène uniquement local puisque les déformations du massif de sol ainsi que celles de l'inclusion interviennent. On peut cependant faire l'hypothèse simplificatrice d'un mécanisme par tranches planes, perpendiculaires à l'inclusion, ce qui revient à supposer que la déformabilité de l'inclusion est sans influence sur le phénomène du frottement.

Le sol sera considéré comme un milieu purement granulaire, doté d'un angle de frottement interne $\varnothing$, et caractérisé avant la rupture par un phénomène de dilatance positive, c'est-à-dire par une augmentation de volume sous l'influence d'un tenseur purement déviatorique (contrainte moyenne $\sigma_{m}$ nulle). 


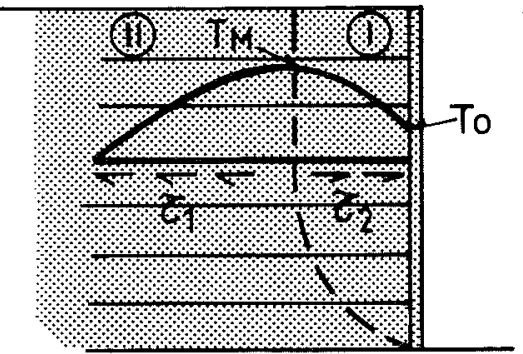

Terre Armée

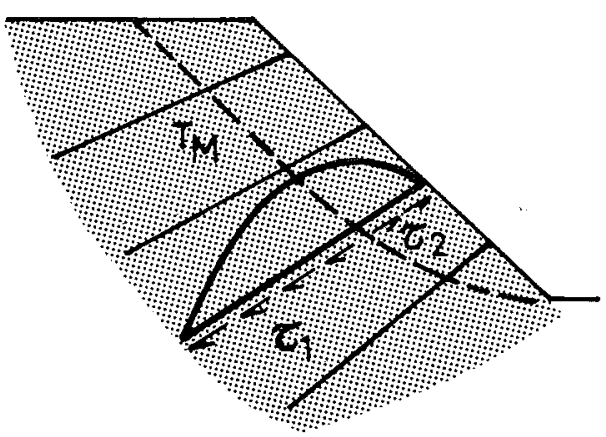

Cloutage

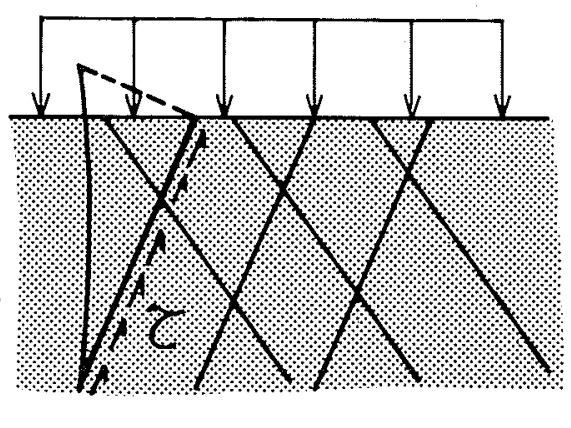

Micropieux

Fig. 2

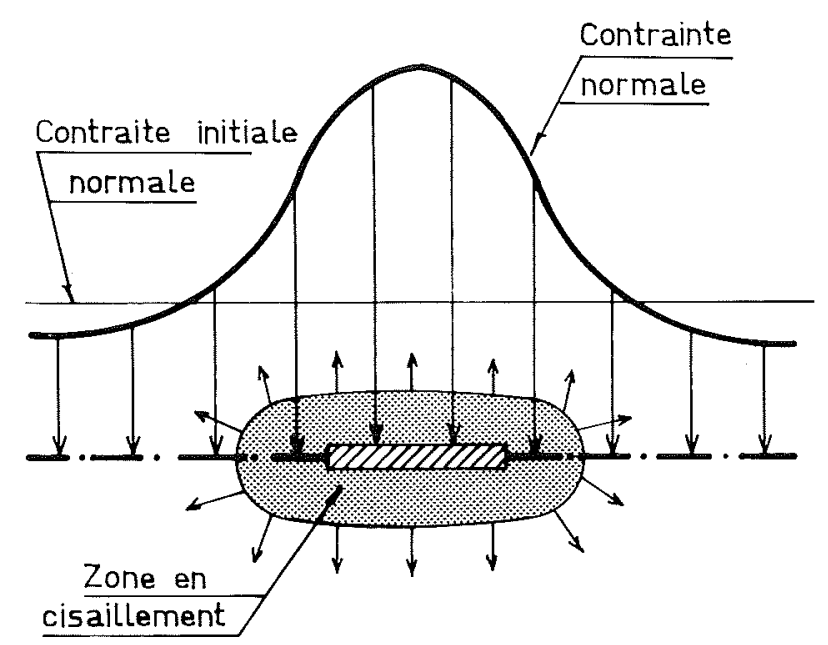

Fig. 3

La dilatance du sol, qui se développe au cours de la mobilisation du frottement sol-inclusion, est de loin le facteur le plus important. Elle intervient de la façon suivante, illustrée à la figure 3 :

La mise en traction (ou en compression) de l'inclusion produit dans le sol des contraintes de cisaillement $\tau$ dont les valeurs ne sont significatives que dans une zone limitée autour de l'inclusion. Dans cette zone le sol a tendance à augmenter de volume par suite de la dilatance, mais s'en trouve en partie empêché par le reste du sol. II en résulte une augmentation importante de la contrainte normale $\sigma$ sur le pourtour de la zone de cisaillement et par suite à la surface de l'inclusion.

Sur le plan théorique et pratique, cette augmentation de la contrainte normale $\sigma$ est difficile à calculer ou à prévoir. Elle est fonction du volume $V_{c}$ de la zone en cisaillement autour de l'inclusion, de la contrainte normale initiale $\sigma_{0}$, de la compressibilité du sol valablement représentée ici par le module pressiométrique $E_{M}$, et des caractéristiques de dilatance $D$, soit :

$$
\Delta \sigma=f\left(V_{c}, \sigma_{0}, E_{M}, D\right) \text { avec } \Delta V_{d}=\frac{\tau_{m}}{D}
$$

En 1975, R. Frank a présenté une étudè théorique de l'influence de la dilatance sur le frottement latéral des pieux. Le comportement du sol était supposé élastique linéaire, mais dilatant, et les calculs ont été faits à partir de la mùthode des éléments finis. Les résultats les plus typiques sont donnés à la figure $4 \mathrm{a}$, mais, contrairement à ce qui vient d'être exposé, l'augmentation de contrainte normale $\Delta \sigma$ n'apparaît sensible qu'à un ou deux rayons à partir de la surface latérale du pieu. Sur la surface même il n'y a guère de différence entre le comportement élastique simple et le comportement élastique dilatant.

A priori ces résultats pourraient être dus au type de modèle de comportement utilisé. En effet l'augmentation de contrainte normale $\Delta \sigma$ a pu être mesurée en dépit des difficultés expérimentales que cela représente. Des premiers résultats ont été obtenus par E. Wernick (1977) dans le cas d'un cylindre vertical muni de cellules de pression et placé au sein d'une cuve remplie de sable. Au cours d'un essai d'arrachement on constate que la valeur de la contrainte normale est multipliée par 10 (fig. 4 b).

Dans beaucoup de cas l'augmentation de la contrainte normale devient ainsi prédominante par rapport à la contrainte initiale $\sigma_{0}$. La valeur du frottement latéral limite est alors pratiquement indépendante de la valeur initiale $\sigma_{0}$ régnant dans le sol avant que ne s'exercent des efforts dans l'inclusion. C'est ce que l'on constate dans la pratique dans les pieux, les tirants et en partie les barres passives, c'est-à-dire pour tous les renforcements in situ. Ainsi pour un pieu, au-delà d'une profondeur critique $z_{c}$, il n'y a pas d'augmentation du frottement latéral limite $\tau_{r}$, en fonction de la profondeur $z$, contrairement à ce qu'indique la formule approximative suivante, parfois encore utilisée:

$$
\begin{aligned}
& \tau_{i}=K_{0}\left(\sigma_{v}\right)_{0} \operatorname{tg} \varnothing_{a} \\
& \tau_{i}=K_{0} \cdot \gamma z \cdot \operatorname{tg} \varnothing_{a}
\end{aligned}
$$

$K_{0}$ : coefficient de pression latérale des terres au repos $\varnothing_{\mathrm{a}}$ : angle de frottement entre le matériau de l'inclusion et le sol

Dans les renforcements de sols in situ, la mise en place de l'inclusion par fonçage ou scellement modifie complètement la valeur de la contrainte initiale normale $\sigma_{0}$ et ne permet même plus ce type de raisonnement.

Compte tenu de la complexité du frottement solinclusion, il est donc nécessaire de préciser clairement à la fois les définitions et les notations. Dans l'hypothèse du comportement par tranches planes, on considèrera la contrainte de cisaillement $\tau$ s'exerçant à l'interface sol-inclusion: il s'agira d'une contrainte moyenne sur la section considérée de l'inclusion. Le coefficient de frottement $\mu$ sera défini par le rapport de 

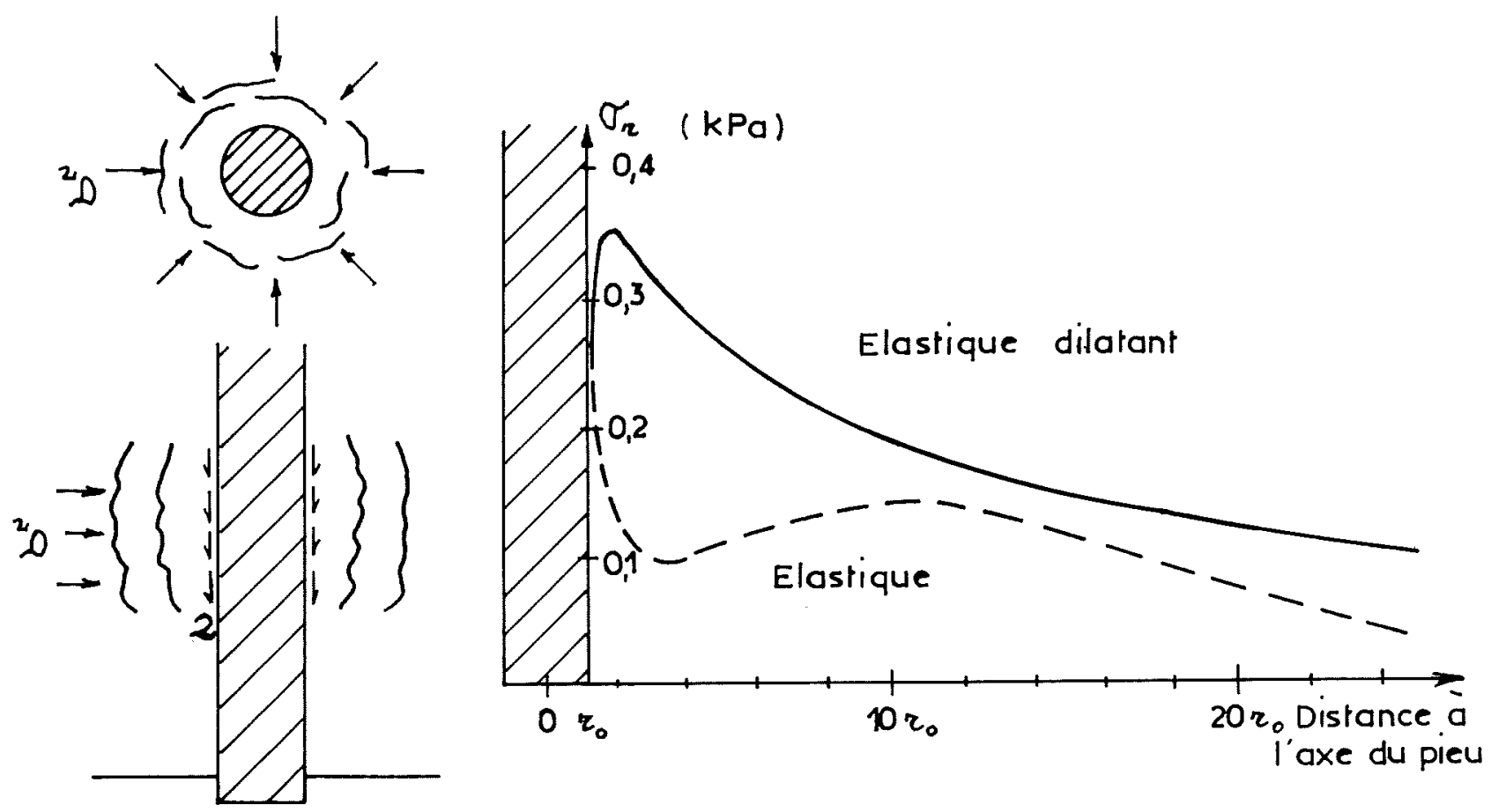

Fig. 4 a

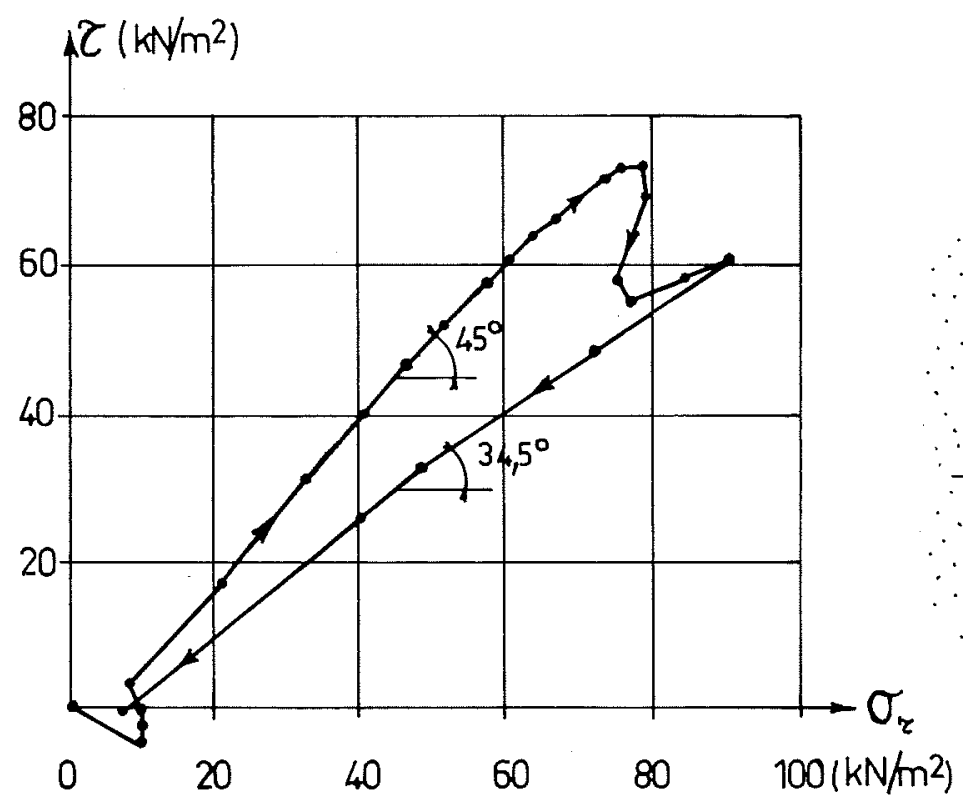

Fig. $4 b$

la contrainte maximale $\tau_{\text {max }}$, appelée frottement latéral unitaire, à la contrainte normale moyenne $\sigma$ correspondante sur la section:

$$
\mu=\frac{\tau_{\max }}{\sigma\left(\tau_{\max }, \sigma_{0}\right)}
$$

II s'agit là d'une définition purement théorique puisque la contrainte normale dépend en fait de la valeur de $\tau_{\max }$, de la contrainte normale initiale $\sigma_{0}$, sans doute également de l'histoire du chargement, et n'est donc généralement pas connue.

Nous ferons par ailleurs l'hypothèse que la valeur de $\tau_{\max }$ n'est fonction que de l'angle de frottement interne $\varnothing$ du sol, de la nature du matériau constituant l'inclusion et de l'état de surface de l'inclusion. Elle sera donc supposée indépendante de la contrainte normale $\sigma$.

\section{3 Étude du coefficient de frottement}

La mesure du coefficient de frottement $\mu$ entre le sol et l'inclusion peut s'effectuer à la boîte de cisaillement en plaçant dans l'une des demi-boîtes une pièce du même matériau que celui de l'inclusion et dans l'autre le sol granulaire à renforcer. 


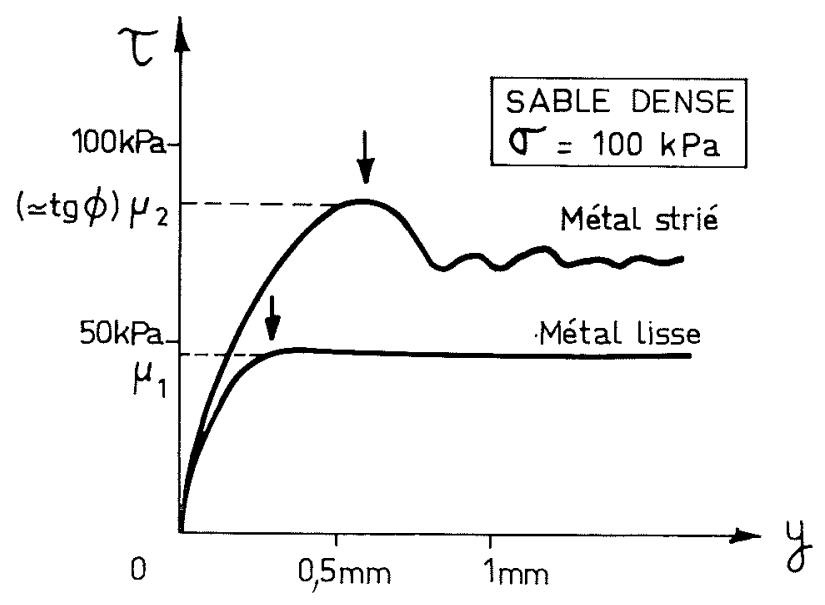

Fig. 5

\subsection{Influence de l'état de surface de l'inclusion}

Les courbes efforts-déplacements de la figure 5 montrent l'influence déterminante de l'état de surface de l'inclusion. Dans le cas d'une surface métallique lisse, la courbe de cisaillement ne présente pas de pic mais un palier situé nettement au-dessous du pic de la courbe de cisaillement sol-sol. L'expérience montre que la formule:

$$
\mu=0,5 \operatorname{tg} \varnothing
$$

constitue une enveloppe inférieure du frottement sol-inclusion (Schlosser et Long, 1974).

Lorsque la surface est rugueuse, soit de façon artificielle par des stries placées perpendiculairement au déplacement, soit de façon naturelle, la courbe de cisaillement présente un pic qui peut très facilement atteindre celui correspondant au frottement interne du sol.

Ce phénomène est lié au mécanisme du frottement sol-inclusion dont les facteurs principaux sont les mêmes que celui du frottement interne, à savoir :

1) Frottement intergranulaire pur.

2) Réarrangement des grains.

3) Dilatance.

La figure 6 tirée des études de Rowe (1962) sur la dilatance montre l'influence respective de ces divers facteurs sur l'angle de frottement interne $\varnothing$.

Lorsque la surface de l'inclusion est lisse, le nombre et le volume des grains mis en déplacement lors du mouvement de l'inclusion sont bien plus faibles que lorsque la surface est rugueuse. La contribution du réarrangement et de la dilatance, fonction de ce volume, se trouve donc sensiblement diminuée. Dans ce cas, la surface de rupture coïncide avec la surface de séparation entre le sol et l'inclusion. Compte tenu de sa parfaite planéité, elle constitue en effet une surface de faible résistance mécanique.

Au contraire, lorsque l'inclusion est rugueuse, elle a tendance à retenir les grains par ses aspérités. II en résulte une augmentation du volume des grains déplacés et donc une augmentation du coefficient de frottement $\mu$. A la limite, lorsque le nombre, la forme et la dimension des aspérités sont suffisantes, le frottement sol-inclusion devient égal au frottement interne du sol. La rupture a alors lieu au sein du sol et non plus au contact de l'inclusion.

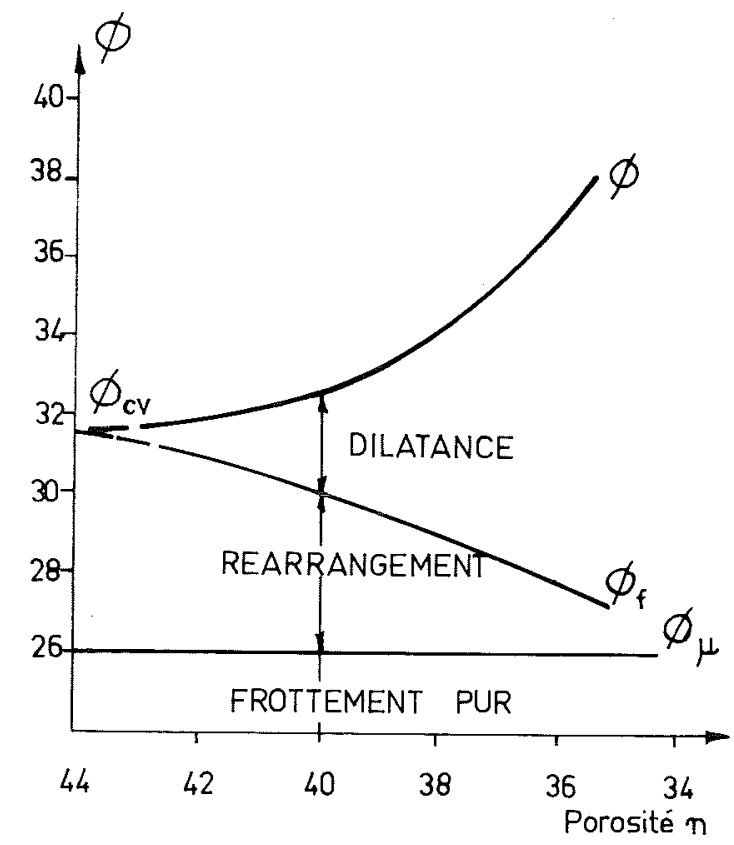

Fig. 6

\subsection{Influence de la contrainte moyenne}

La formule de la dilatance de Rowe (1962), établie à partir des résultats d'essais triaxiaux, montre l'influence de l'augmentation du volume. $\left(\frac{d v}{d \varepsilon_{\mathrm{a}}}\right)$ sur l'angle de frottement interne. Elle s'écrit en effet :

$$
\frac{\sigma_{\mathrm{a}}}{\sigma_{\mathrm{r}}}=\left(1+\frac{\mathrm{dv}}{\mathrm{d} \varepsilon_{\mathrm{a}}}\right) \operatorname{tg}^{2}\left(\frac{\pi}{4}+\frac{\varnothing_{\mathrm{t}}}{2}\right) \quad \begin{aligned}
& \sigma_{\mathrm{a}}: \text { contrainte axiale } \\
& \sigma_{\mathrm{r}}: \text { contrainte radiale }
\end{aligned}
$$

où $\varnothing_{f}$ est l'angle de frottement résultant du frottement grain sur grain $\varnothing_{\mu}$ et du réarrangement.

Les valeurs de $\varnothing_{\mathrm{f}}$ et de $\frac{d v}{d \varepsilon_{\mathrm{a}}}$ dépendent de l'indice des vides initial $e_{o}$, c'est-à-dire de la compacité du milieu granulaire. Ils varient en sens inverse, l'angle $\varnothing_{\mathrm{f}}$ diminuant avec l'indice des vides, alors que la dilatance augmente avec la compacité.

Mais il existe un autre facteur qui, toutes choses égales par ailleurs, influe sur la valeur de l'angle de frottement interne $\varnothing$. C'est la contrainte moyenne $\sigma_{m}$. La figure 7 montre cette influence: les courbes intrinsèques des sols pulvérulents ne sont pas entièrement des droites, mais ont une allure parabolique pour les faibles contraintes moyennes. Le coude peut se situer à des valeurs très variables: $25 \mathrm{kPa}$ pour un sable à granulométrie uniforme, $150 \mathrm{kPa}$ pour une granulométrie très étalée. Ce phénomène a été mis en évidence par Bishop (1966). II conduit à la définition et à l'utilisation d'une cohésion apparente $c_{a}$ fonction, comme l'angle de frottement interne $\varnothing$, du niveau de la contrainte moyenne $\sigma_{m}$. Dans les modèles réduits, il agit comme un effet d'échelle rendant encore plus difficile l'application des lois de la similitude. Dans les projets réels il est le plus souvent négligé mais il doit être pris en compte dans les sols compacts à granulométrie très étalée. 


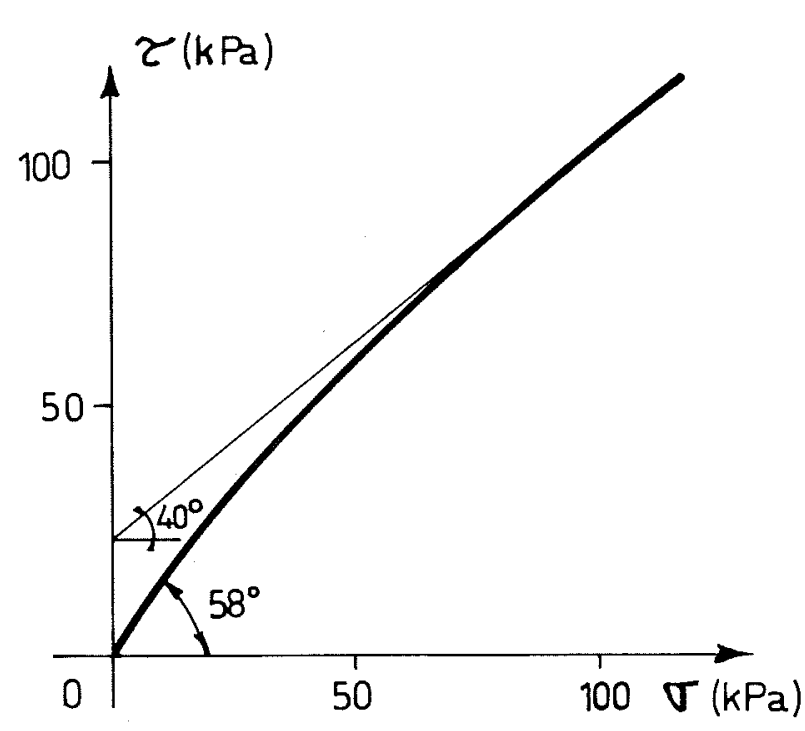

SABLE FIN

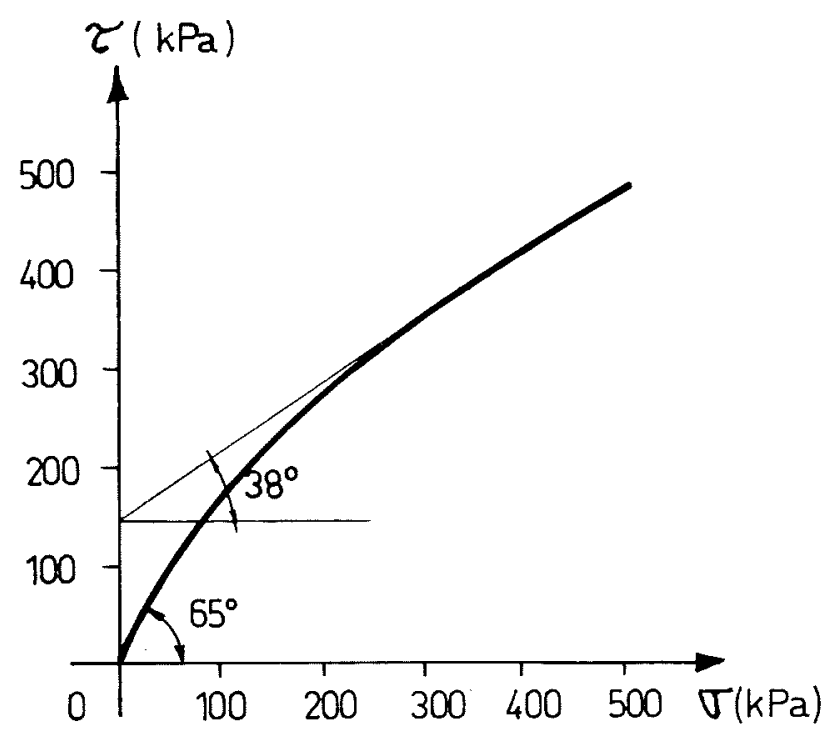

MORAINE COMPACTE

Fig. 7

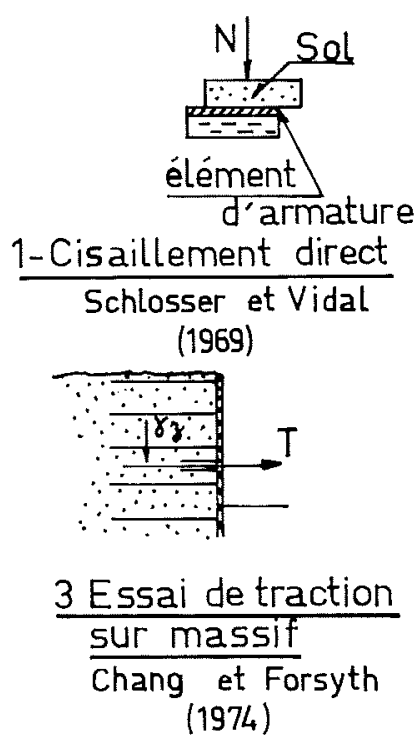

Fig. 8
Ce phénomène est en grande partie lié à la dilatance du sol. Celle-ci diminue en effet non seulement lorsque l'indice des vides initial $e_{0}$ augmente, mais également lorsque la contrainte moyenne $\sigma_{m}$ augmente. L'angle $\varnothing_{\text {f }}$ par contre reste indépendant de $\sigma_{\mathrm{m}}$. $\|$ en résulte ainsi d'après la formule de Rowe une augmentation de la valeur de $\varnothing$ aux faibles valeurs de $\sigma_{m}$.

\section{Mesure du frottement : frottement latéral unitaire et coefficient de frottement apparent}

\subsection{Généralités}

L'effet tridimensionnel et l'influence de la dilatance sont difficiles à prendre en compte dans un calcul pratique en utilisant la donnée initiale du coefficient $\mu$. C'est la raison pour laquelle plusieurs méthodes ont été développées pour pouvoir mesurer des paramètres plus globaux que ce coefficient de frottement élémentaire $\mu$. La figure 8 montre par exemple quatre méthodes de mesure du frottement envisageables dans le cas de la terre armée:

1) l'essai classique à la boîte de cisaillement,

2) l'essai de traction à la grande boîte,

3) l'essai de traction ou d'arrachement au sein d'un massif de sol,

4) l'essai de rotation sur modèle de mur en terre armée.

L'essai de frottement le plus représentatif est sans doute celui qui consiste à mesurer le frottement sur l'ensemble des armatures du massif en terre armée, c'est-à-dire à construire un modèle de mur en terre armée et à le déformer par exemple en rotation comme dans la méthode $n^{\circ} 4$. Cet essai se heurte à plusieurs difficultés: problèmes de similitude, coût et durée importants. Dans la pratique la plupart des mesures de frottement sont effectuées par des essais d'arrachement de l'inclusion qui présentent notamment l'avantage de tenir compte de la mise en place de l'inclusion. 


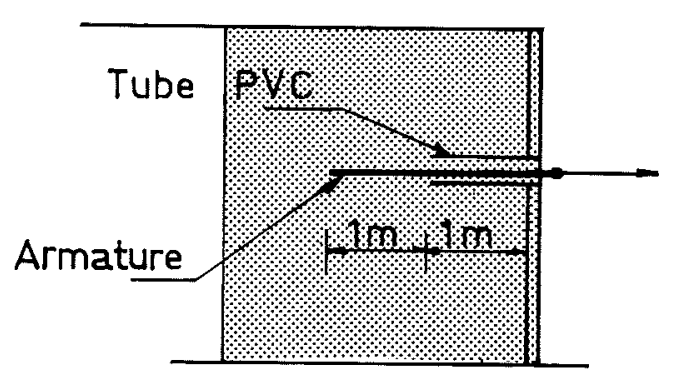

Terre Armée

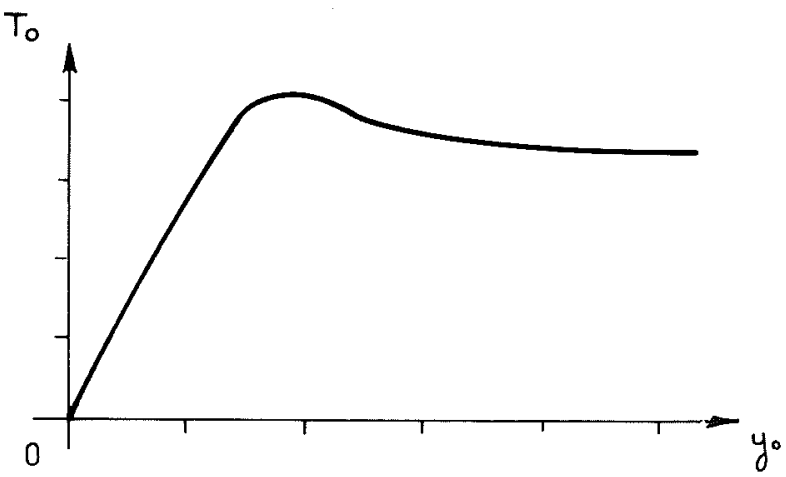

Fig: 10

\subsection{L'essai d'arrachement d'une inclusion : technologie et principe}

L'essai consiste à placer dans le sol une inclusion, suivant le même mode de mise en place que celui utilisé dans la technique de renforcement considérée.

Afin d'éviter les effets de bord, l'inclusion est placée sous gaine dans sa partie située près de la surface du sol, ce qui annule tout frottement dans cette zone. La figure 9 montre schématiquement un tel dispositif dans la terre armée d'une part, dans le cloutage ou les micropieux d'autre part.

L'essai est réalisé en tirant sur l'inclusion avec une vitesse de déplacement la plus constante possible jusqu'à obtenir son arrachement du sol. II convient d'éviter la rupture de l'inclusion par traction; il faut donc limiter sa longueur dans la partie proprement résistante, ce qui nécessite un prédimensionnement correct de cette longueur. Dans la pratique les longueurs résistantes sont de 1 à $2 \mathrm{~m}$.

L'effort de traction est exercé en tête de l'inclusion par vérinage en prenant appui à la surface du sol.

Le résultat de l'essai est donné sous la forme d'une courbe efforts en tête $T_{0}$ - déplacements en tête $Y_{0}$.

L'essai d'arrachement d'une inclusion présente donc une certaine analogie avec l'essai d'arrachement d'un pieu ou avec l'essai de chargement statique d'un pieu à condition de négliger l'effort de pointe. II en diffère cependant par le fait qu'il s'agit d'un essai à déformation contrôlée contrairement à l'essai de chargement statique où la charge est appliquée par paliers. On peut donc y mettre en évidence des efforts de pic et des efforts résiduels.

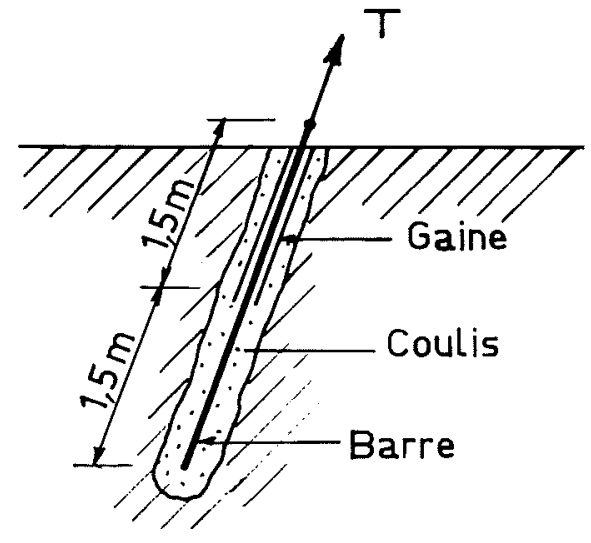

Clous - Micropieux

\subsection{Coefficient de frottement apparent}

Dans la technique de la terre armée, la contrainte normale initiale $\sigma_{0}$, s'exerçant à la surface de l'inclusion à extraire est assez bien connue. Les armatures sont en effet des bandes métalliques placées horizontalement au fur et à mesure de la construction du remblai armé. La contrainte $\sigma_{0}$ est donc voisine de la pression due au poids des terres, soit :

$$
\sigma_{\mathrm{o}} \simeq \gamma z
$$

C'est la raison pour laquelle on définit dans l'essai d'arrachement d'une inclusion un coefficient de frottement apparent $\mu^{*}$ entre le sol et l'armature (Alimi et al. 1977) par la formule :

$$
\mu^{\star}=\frac{\tau_{\max }}{\sigma_{0}}
$$

où $\tau_{\max }$ est la contrainte de cisaillement moyenne sur les faces de l'armature au moment de l'arrachement.

Dans la mesure où l'on peut supposer que l'armature a un comportement rigide par rapport au sol, la valeur maximale $T_{\max }$ de l'effort de traction en tête correspond à une mobilisation complète du frottement tout au long de l'armature. On tire alors la valeur de $\mu^{*}$ de la mesure de $T_{\max }$ par l'expression:

$$
\mu^{*}=\frac{T_{\max }}{2 b L \gamma z}
$$

où b est la largeur de l'armature et $L$ la longueur résistante de l'armature.

Les valeurs obtenues pour le coefficient de frottement apparent $\mu^{*}$ peuvent être beaucoup plus grandes que le coefficient de frottement réel $\mu$. On a en effet :

$$
\mu^{*}=\frac{T_{\text {max }}}{\sigma_{0}} \geqslant \mu=\frac{T_{\max }}{\sigma_{0}+\Delta \sigma\left(\tau_{\max }\right)}
$$

où $\Delta \sigma$ est le supplément de contrainte normale due au phénomène de dilatance. II est courant d'obtenir des valeurs très supérieures à 1 pour les faibles valeurs de $\sigma_{0}$.

La valeur de $\mu^{\star}$ n'est pas constante. Elle dépend en effet de $\sigma_{0}$ et donc de la profondeur z. Comme la dilatance, elle diminue lorsque la valeur de $\sigma_{0}$ augmente. Aux fortes profondeurs $\mu^{\star}$ devient voisin de $\mu$. 


Essais sur bandes métalliques
enterrées dans du sable à
différentes densités relatives

Largeur des bandes: $1,5 \mathrm{~cm}$

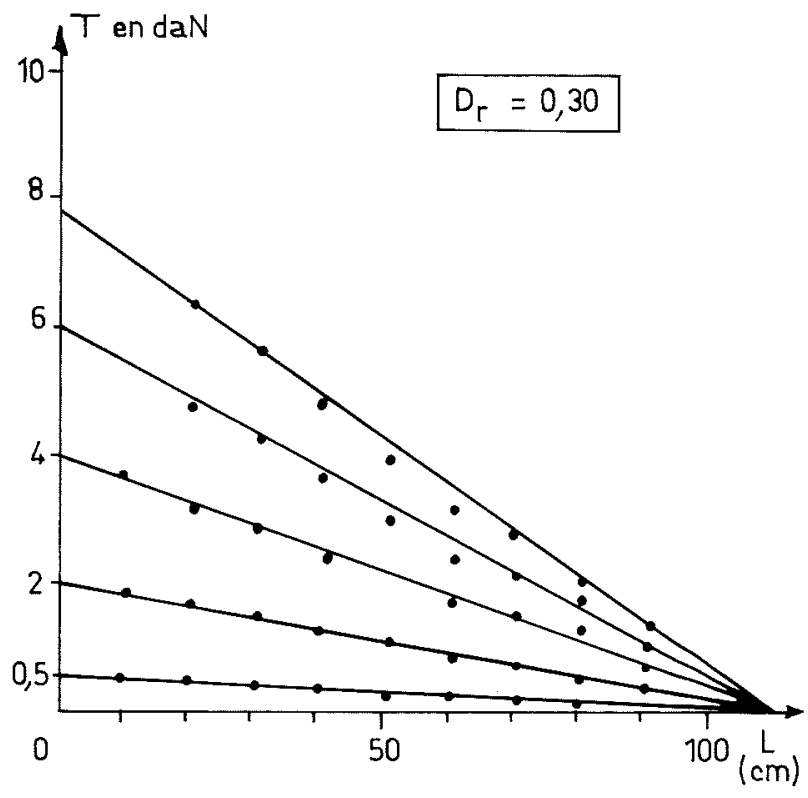

\subsection{Frottement latéral unitaire}

Dans les techniques du cloutage et des micropieux, la contrainte initiale normale $\sigma_{0}$ sur l'inclusion est difficile à évaluer. Elle est principalement fonction de la mise en place de l'inclusion: scellement dans un forage préalable ou fonçage.

Le forage réduit à zéro la contrainte normale qui régnait au sein du sol. Après mise en place de la barre et injection du coulis, la contrainte normale initiale $\sigma_{0}$ est essentiellement fonction de la pression d'injection et des différentes passes d'injection. Sa valeur peut varier dans une assez large gamme, mais il n'y a pas encore à l'heure actuelle de mesures précises dans ce domaine.

Le fonçage augmente les efforts normaux initiaux de deux façons : d'abord par refoulement du sol, puis par phénomène de dilatance associé au frottement latéral. A la fin du fonçage il ne reste que l'influence du refoulement, car l'effet de la dilatance est réversible (Wernick, 1977). Dans le temps l'effet du refoulement s'atténue, mais la contrainte initiale normale $\sigma_{0}$ reste supérieure à ce qu'elle était au sein du sol.

$\mathrm{Ne}$ pouvant ainsi pas connaître la valeur de la contrainte initiale $\sigma_{0}$, on caractérise le frottement sol-inclusion par la valeur du frottement latéral unitaire $\tau_{\max }$. La valeur de $\tau_{\max }$ intègre les conditions de mise en place, l'état de contrainte initiale dans le sol et la géométrie de l'inclusion.

Dans le cas d'une barre scellée, l'expérience montre que dans un essai d'arrachement la barre et le coulis se

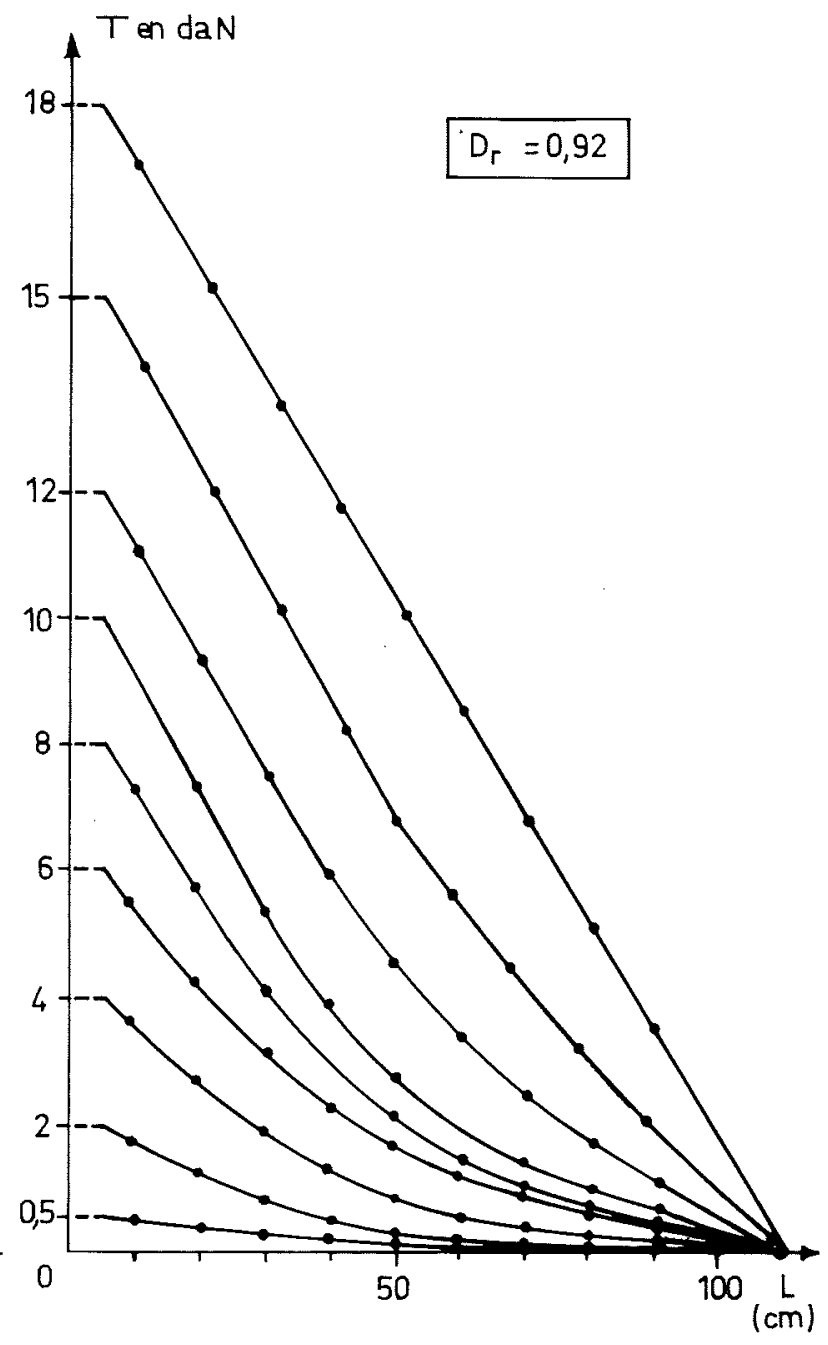

Fig. 11

comportent comme une inclusion unique et que la rupture a lieu entre le coulis et le sol, approximativement sur la surface du forage. Cet aspect doit être pris en compte dans le calcul de $\tau_{\max }$ qui se fait par la formule :

$$
\tau_{\max }=\frac{T_{\max }}{S \cdot L}
$$

où : $S$ est l'aire latérale de la surface de rupture $L$ la longueur résistante de l'inclusion.

La détermination de $\tau_{\max }$ se heurte à l'estimation de la surface latérale, laquelle est particulièrement difficile lorsque le coulis est injecté sous une forte pression. L'expérience montre en effet que dans ce cas, il y a, outre une augmentation du diamètre initial du forage, des poches parfois importantes dans les zones de faible résistance du sol, ainsi que des phénomènes de claquage lorsque la pression d'injection est forte.

\section{Influence de la déformabilité de l'inclusion}

\subsection{Résultats expérimentaux}

De nombreux essais de traction de bandes métalliques résistantes et peu épaisses ont été réalisés en modèle réduit en mesurant la distribution de l'effort normal tout au long de l'inclusion (Alimi, 1977). La figure 11 montre un résultat caractéristique où l'effet de la déformabilité relative de l'inclusion est bien marqué (pour une même inclusion, la déformabilité relative varie dans le même sens que la densité relative du sol). 
A forte déformabilité relative, pour de faibles efforts de traction en tête, seule une partie de l'inclusion est sollicitée. La forme de la courbe de distribution des tractions montre qu'il y a une mobilisation progressive du frottement à partir de la tête de l'inclusion. Au fur et à mesure que l'effort en tête augmente, il y a saturation progressive de ce frottement, pour aboutir à une mobilisation complète correspondant à une distribution linéaire des tractions au moment de l'arrachement. La longueur de bande sollicitée, appelée longueur efficace, apparaît principalement fonction de la rigidité de l'inclusion, c'est-à-dire de sa section et du module de déformation du matériau qui la constitue, ainsi que de la loi élémentaire du frottement sol-inclusion.

Ce type de saturation progressive n'est pas un phénomène nouveau. II a été mis en évidence depuis longtemps dans le frottement latéral des pieux (Dinesh Mohan, 1963, et Cambefort, 1964).

\subsection{Modèle théorique}

Il est possible de développer un modèle à partir d'une loi élémentaire simple pour le frottement sol-inclusion. Nous prendrons le cas d'une loi élastoplastique sans écrouissage. Un calcul similaire a été développé pour les pieux par Baguelin et Venon (1971). Ces auteurs ont pris en compte dans la loi élémentaire du frottement une ordonnée à l'origine dont l'influence est fondamentale dans la détermination de la longueur efficace sur laquelle s'exerce le frottement.

Si $y(x)$ est le déplacement d'un point de l'inclusion situé à l'abscisse $x$, les équations permettant de calculer $y(x)$ sont au nombre de deux : d'une part l'équilibre local d'un petit élément de longueur $d x$, d'autre part l'équation de comportement élastique de cet élément d'inclusion.

On obtient :
$T(y)=\frac{1}{P} \frac{d T}{d x}$
(1) Équilibre local
$d y=\frac{1}{E S} T d x$
(2) Allongement élastique

$P$ : périmètre d'une section droite de l'inclusion

avec: $S$ : section de l'inclusion

$E$ : module de déformation du matériau constitutif

L'élimination de la traction $T$ conduit à l'équation :

$$
\frac{d^{2} y}{d x^{2}}=\frac{P}{E S} \tau(y)
$$

où $\tau(y)$, dont la valeur est ici constamment négative, est donné par la loi élémentaire du frottement, soit :

$$
\begin{array}{lll}
\tau(y)=k y & \text { pour } & |y|<\frac{\tau_{\max }}{k} \\
\tau(y)=-\tau_{\max } & \text { pour } & |y| \geqslant \frac{\tau_{\max }}{k}
\end{array}
$$

Le coefficient $k$ est l'analogue d'un coefficient de réaction et $\tau_{\max }$ le frottement latéral unitaire.

Dans un premier temps, il est utile et commode de considérer le cas de l'inclusion de longueur infinie. La résolution de l'équation différentielle précédente donne les expressions suivantes:

$$
y=-\frac{P \tau_{\max }}{E S} \frac{x^{2}}{2}+\frac{T_{0}}{E S} x+y_{0} \text { pour }|y| \geqslant \frac{\tau_{\max }}{k}
$$
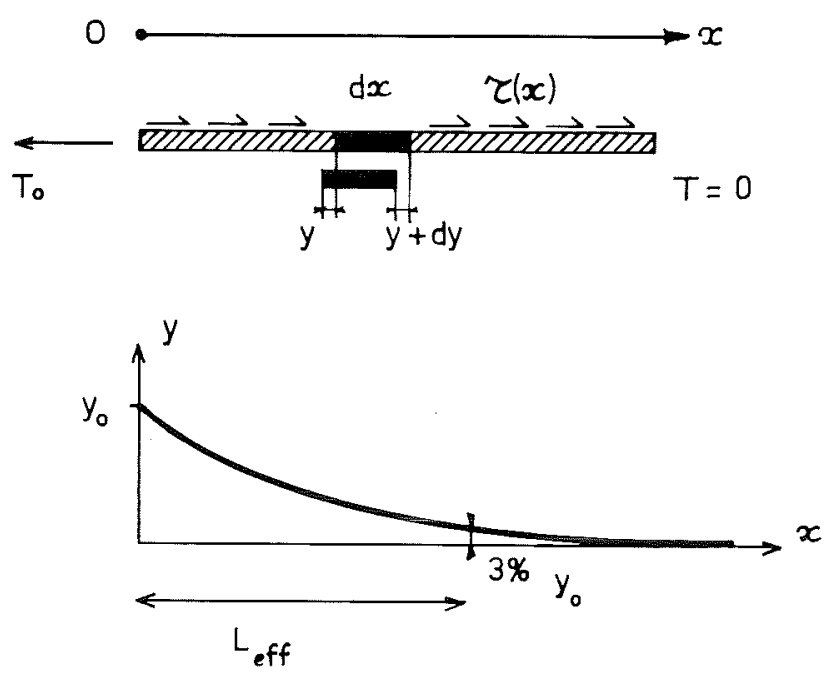

Fig. 12

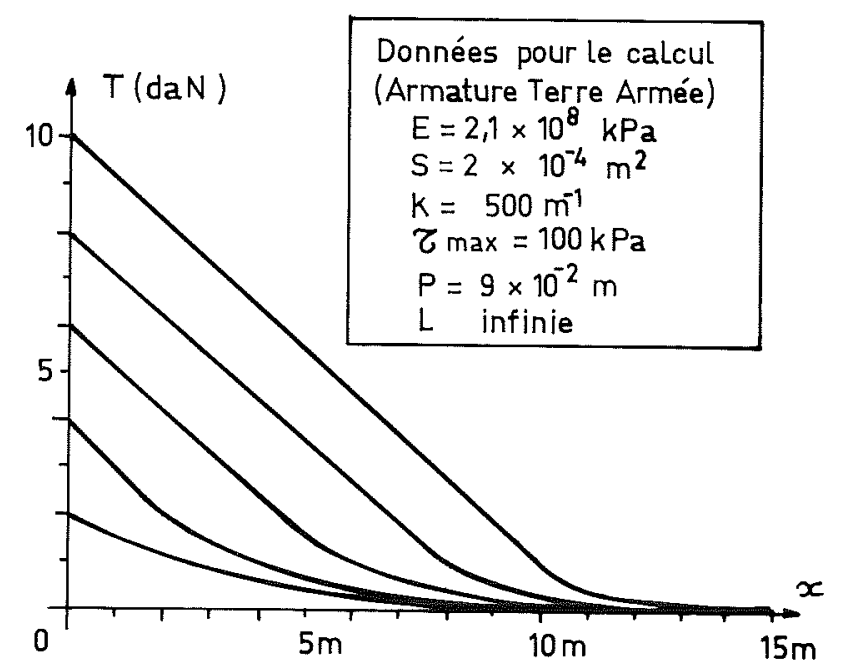

Fig. 13

$$
y=-\frac{\lambda T_{0}}{E S} e^{-\frac{x}{\lambda}} \quad \text { pour }|y| \leqslant \frac{\tau_{\max }}{k}
$$

où $\lambda=\sqrt{\frac{\mathrm{ES}}{\mathrm{kP}}}$ est appelée longueur efficace de référence. Les conditions aux limites sont les suivantes :

$$
\begin{array}{ll}
x \text { infini } & y^{\prime}=\frac{T}{E S}=0 \\
y=0 & \\
x=0 & y^{\prime}=\frac{T_{0}}{E S}
\end{array}
$$

Ces deux expressions se raccordent pour $|y|=\frac{\tau_{\max }}{k}$. Elles permettent par dérivation de calculer la distribution des tractions représentée à la figure 13 pour diverses valeurs de $T_{0}$ et pour une longueur $L$ infinie. On remarque que, dans la zone de saturation du frottement, la distribution des tractions est linéaire avec la même pente pour toutes les courbes. 
Contrairement à ce que l'on observait sur les courbes expérimentales, la traction reste finie et tend asymptotiquement vers zéro avec $x$. On ne peut donc pas mettre en évidence rigoureusement de longueur efficace. Cependant la traction T et le déplacement y deviennent très faibles et pratiquement négligeables dès que l'on dépasse une certaine longueur. C'est cette longueur que nous définirons comme longueur efficace, en considérant par exemple un pourcentage du déplacement en tête $y_{0}$ arbitrairement fixé à la valeur de $3 \%$.

La longueur efficace est alors donnée par:

$$
L_{\text {eff }}=-\lambda \operatorname{Ln} 0,03 \text { soit } L_{\text {eff }}=3,5 \lambda
$$

Elle est ainsi directement proportionnelle à la longueur efficace de référence et ne dépend en particulier pas de l'effort de traction en tête $\mathrm{T}_{\mathrm{o}}$

Si l'on considère alors une inclusion de longueur $\mathrm{L}$ finie, les nouvelles conditions aux limites $\left(T=T_{0}\right.$ pour $y=y_{0} ; T=0$ pour $y=L$ ) permettent d'obtenir l'expression suivante de $\mathrm{L}_{\text {eff }}$ :

$$
L_{\text {eff }}=L-\lambda \operatorname{Arg} \operatorname{ch}\left[0,03 \operatorname{sh} \frac{L}{\lambda}\right]
$$

qui montre qu'il n'y a existence d'une longueur efficace que pour des valeurs suffisamment grandes de $L$, et en particulier plus grandes que la valeur $3,5 \lambda$ déterminée précédemment dans le cas de l'inclusion de longueur infinie. Cela s'explique par le fait que la rigidité relative de l'inclusion est, toutes choses égales par ailleurs, d'autant plus grande que la longueur est plus faible. A la limite, une inclusion très courte se comporte comme un élément rigide avec mobilisation uniforme du frottement.

Les calculs montrent que la longueur efficace dans le cas d'une longueur d'inclusion finie est en fait très peu dépendante de la longueur $L$ et très voisine de la valeur $3,5 \lambda$ (variation relative inférieure à $10 \%$ dès que la longueur L est supérieure de $20 \%$ à la longueur efficace $3,5 \lambda$ ).

\section{Paramètres influençant $\mu^{*}$ et $\tau_{\max }$}

Le coefficient de frottement apparent $\mu^{*}$ et le frottement latéral unitaire $\tau_{\max }$ dépendent bien sûr de l'angle de frottement interne $\varnothing$ du milieu granulaire considéré. L'expérience a cependant mis en évidence trois paramètres principaux dont l'influence s'avère prépondérante sur le frottement sol-inclusion, à savoir :

- la compacité du milieu granulaire,

- l'état de surface de l'inclusion,

- la contrainte normale initiale sur l'inclusion.

\subsection{Influence de la compacité du sol}

La figure 14 montre l'influence de la compacité sur la courbe de mobilisation du coefficient de frottement apparent $\mu^{*}$ au cours d'essais d'extraction de bandes métalliques lisses en modèle réduit. Aux faibles densités, le pic est obtenu pour un faible déplacement (quelques millimètres) et il est très accentué. La valeur résiduelle de $\mu^{*}$ est inférieure de moitié à celle correspondant au pic. Aux fortes densités, le pic s'efface au profit d'un palier obtenu pour de grands déplacements (plusieurs centimètres).

Si les valeurs maximales de $\mu^{*}$ correspondant aux faibles densités restent assez voisines du frottement réel $\mu$ mesuré à la boîte de cisaillement, il n'en est plus

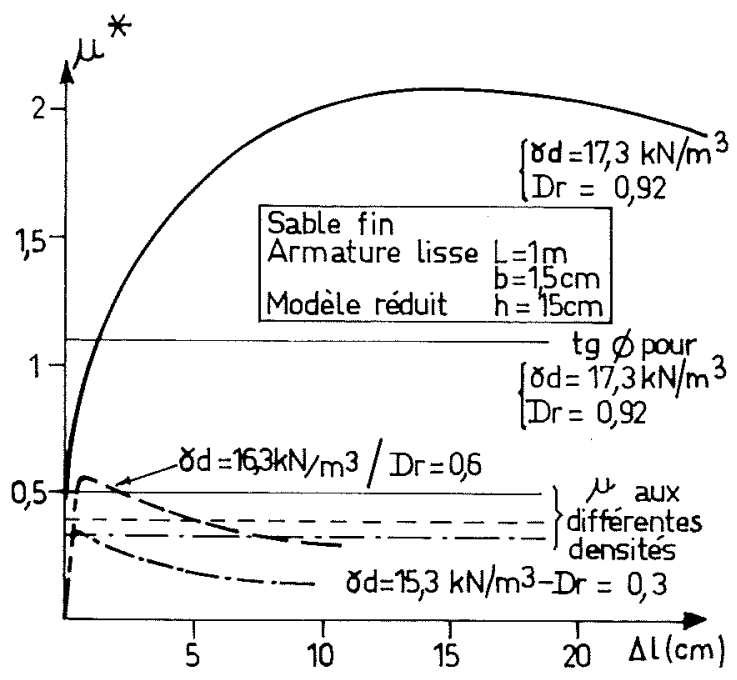

Fig. 14

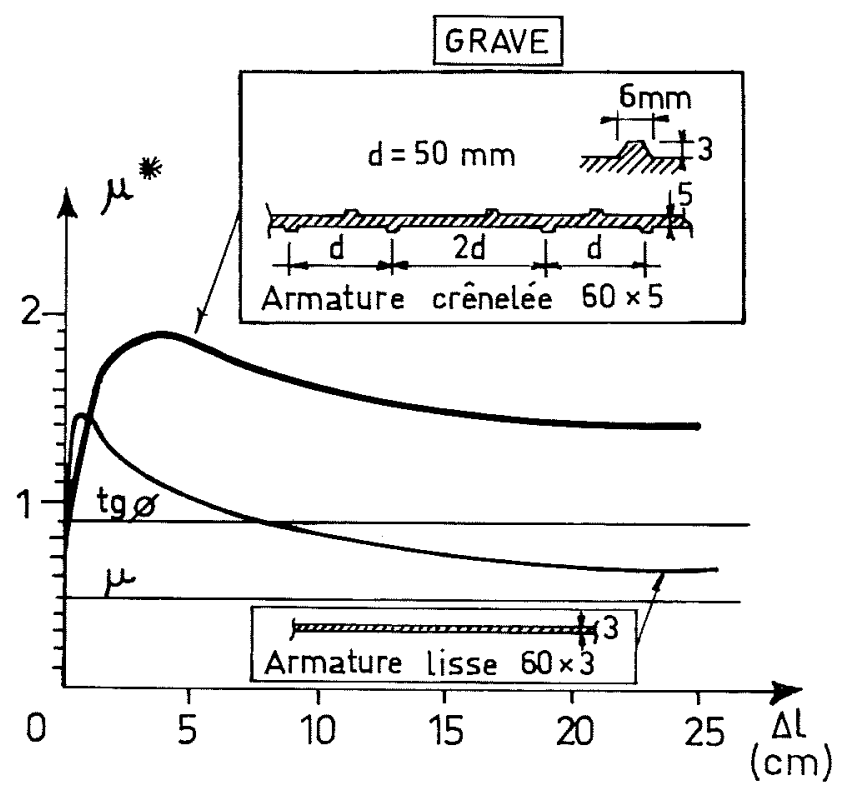

Fig. 15

de même du frottement apparent aux fortes densités, où les valeurs de $\mu^{*}$ sont fortement supérieures à 1 .

On peut expliquer ces résultats par le fait qu'à forte densité, le sol granulaire possède une dilatance positive, et que les efforts de cisaillement exercés par l'inclusion sur le sol ont tendance à augmenter la contrainte normale qui s'exerce sur sa surface latérale. A faible densité au contraire, il se produit dès les premiers déplacemenits un écroulement local et limité de la structure granulaire avec mobilisation d'effets de voûte, de telle sorte que l'inclusion se déplace comme dans un tunnel avec des contraintes normales très faibles. II y a ainsi une grande différence entre les valeurs de pic et les valeurs résiduelles qui sont bien inférieures au coefficient de frottement réel.

\subsection{Influence de l'état de surface de l'inclusion}

La figure 15 montre les courbes de mobilisation du coefficient de frottement apparent $\mu^{*}$ lors d'essais d'extraction d'armatures lisses et nervurées dans des ouvrages en terre armée. Les principales observations sont les suivantes: 


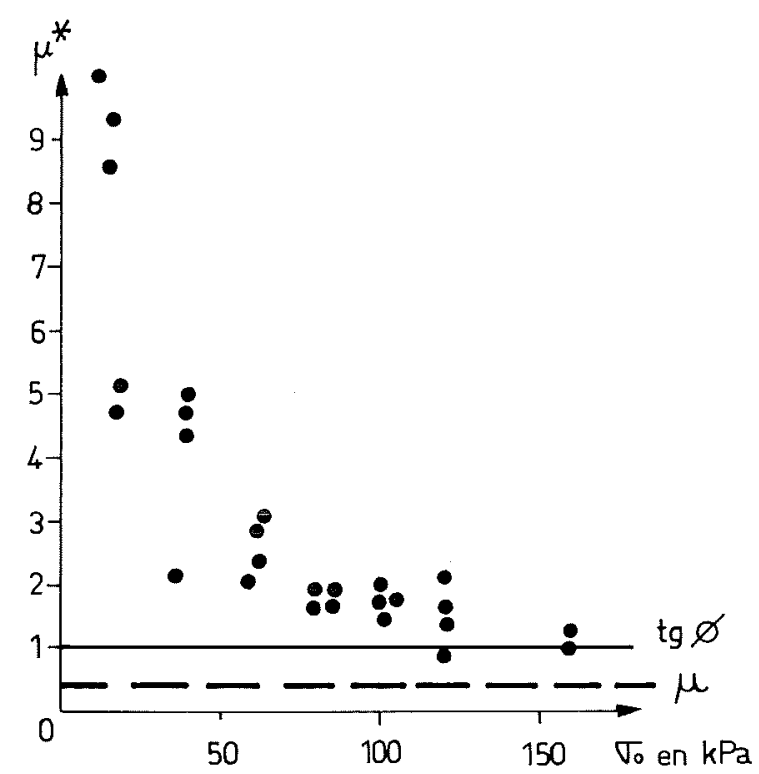

Fig. 16

- la valeur du coefficient de frottement apparent est plus élevée pour des armatures crénelées que pour des armatures lisses,

- le pic est atteint pour des déplacements de l'ordre de $0,5 \mathrm{~mm}$ pour les armatures lisses et de $5 \mathrm{~mm}$ pour les armatures crénelées,

- le pic est très accentué pour les armatures lisses et la valeur résiduelle du frottement est de l'ordre de $50 \%$ de celle du pic. Pour les armatures crénelées, la valeur résiduelle est au contraire peu inférieure à celle du pic.

La différence de comportement entre ces deux types d'armatures provient de la présence des reliefs qui mettent en déplacement un volume de sol plus important que dans le cas d'une surface lisse. Le cisaillement accompagné de dilatance s'exerce donc sur un plus grand volume, ce qui conduit à une augmentation d'autant plus forte de la contrainte normale au voisinage de l'armature. La présence de reliefs force en outre la surface de rupture à se développer au sein du milieu granulaire, faisant ainsi passer le coefficient de frottement réel de $\mu$ à $\operatorname{tg} \varnothing$.

\subsection{Influence de la contrainte normale initiale $\sigma_{0}$}

La figure 16 montre les variations du coefficient de frottement apparent $\mu^{*}$ lors d'essais d'extraction d'armatures crénelées dans des ouvrages en terre armée à différentes profondeurs, et par suite à différentes valeurs de la contrainte normale initiale $\sigma_{0}$ s'exerçant sur l'armature. Le sol granulaire est une grave compactée ayant un angle de frottement interne de $46^{\circ}$ et un angle de frottement sol-acier galvanisé de $27,5^{\circ}$.

On observe que :

le coefficient de frottement apparent diminue quand la contrainte normale augmente,

$\mu^{*}$ atteint un palier voisin de $\operatorname{tg} \varnothing$ pour une contrainte d'environ 100 à $150 \mathrm{kPa}$,

les valeurs de $\mu^{*}$ correspondant à des faibles valeurs de $\sigma_{0}$ sont très élevées (5 à 10 ).
Ces résultats ont été confirmés par des. essais d'extraction d'inclusion effectués dans une grande boîte de cisaillement $(91,5 \times 91,5 \times 46 \mathrm{~cm})$ (Schlosser et Elias, 1978).

Là encore ce résultat s'explique par la dilatance positive des sols granulaires denses. On retrouve le phénomène connu de la diminution de la dilatance quand la contrainte moyenne augmente (Lee et Seed, 1976).

\section{7 Étude de l'effet de la dilatance}

\subsection{Essais de cisaillement à volume constant}

Les résultats exposés dans le paragraphe précédent montrent que la dilatance est le phénomène fondamental intervenant dans le frottement entre une inclusion et un sol granulaire dense.

L'essai de cisaillement direct à la boîte ne permet pas de mettre en évidence et de mesurer l'influence de cette dilatance telle qu'elle s'exerce dans le volume de sol cisaillé entourant l'inclusion, et dont la déformation est contenue par les couches de sol environnantes. L'essai de cisaillement à volume constant, dans lequel on empêche toute variation de volume au cours du cisaillement, représente par contre le cas extrême où l'influence de la dilatance est maximale. Le comportement réel, résultat de la compressibilité du sol, est situé entre ces deux essais; il s'avère en fait assez voisin de l'essai à volume constant.

Guilloux et al. (1979) ont interprété les résultats d'essais à volume constant effectués par Alimi (1978). La boîte de cisaillement était modifiée par un asservissement de la contrainte normale aux variations de volume : un capteur de déplacement contrôlait la hauteur de l'échantillon et déclenchait un dispositif augmentant la contrainte normale quand l'échantillon se dilatait et la diminuant quand il tassait. L'essai à volume constant consiste à enregistrer simultanément les déformations $\varepsilon=\frac{\Delta \ell}{\ell}$ et les contraintes tangentielles $\tau$ et normale $\sigma$ dans le plan de cisaillement.

Deux types d'essais à volume constant ont été réalisés : des essais de cisaillement sable sur sable et des essais de cisaillement sable sur plaque métallique lisse. Les résultats sont présentés à la figure 17 sous deux formes :

1) les courbes de cisaillement $(\tau, \varepsilon)$ où sont indiquées les valeurs successives de la contrainte normale $\sigma$ au cours de l'essai,

2) les chemins de contraintes $(\tau, \sigma)$.

Dans le cisaillement à volume constant sable sur sable, la courbe de cisaillement présente un pic, mais moins accentué que dans un essai à contrainte normale constante. Le chemin des contraintes $(\tau, \sigma)$ montre que le frottement maximal est mobilisé avant ce pic et qu'il correspond dans le cas du sable de Fontainebleau testé à un angle de frottement interne de $34^{\circ}$, en fait bien inférieur à la valeur de l'angle de frottement interne de $43^{\circ}$ déterminée dans un essai de cisaillement à contrainte normale constante. Cette différence s'explique lorsqu'on examine les diverses composantes du frottement interne et les angles $\varnothing_{\mu}$, $\varnothing_{\mathrm{f}}$ et $\varnothing$ (cf. $\S 3.1$ ). L'angle de $34^{\circ}$ correspond en effet à une valeur très acceptable pour $\varnothing_{\mathrm{t}}$, puisqu'elle conduit à un 


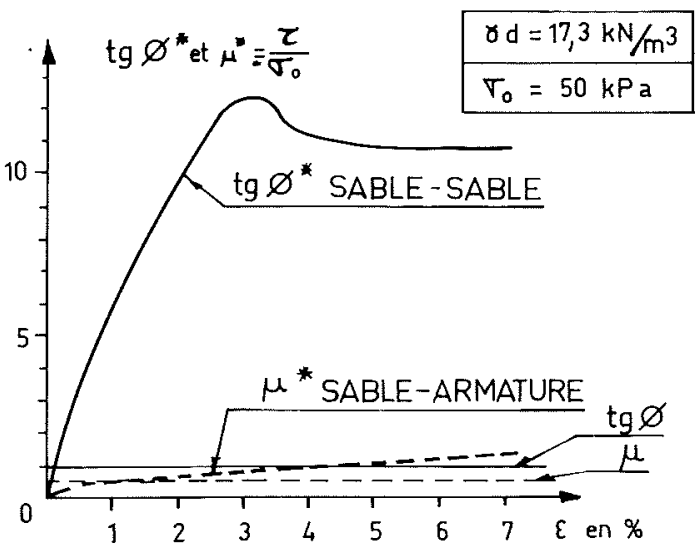

Fig. 18

le coefficient $\operatorname{tg} \varnothing^{\star}$ augmente régulièrement et atteint des valeurs très élevées de l'ordre de 12 ,

le coefficient $\mu^{*}$ augmente beaucoup moins et atteint des valeurs qui ne sont que peu supérieures à $\mu$.

Ces résultats prouvent que dans ce cas, l'influence de la dilatance est très importante dans le frottement sable sur sable. Par contre, elle est minime dans le cisaillement sable sur plaque métallique, où la zone en rupture est de très faible épaisseur (de l'ordre du diamètre des grains) et peut être confondue avec un plan.

La figure 19 montre l'influence de la contrainte initiale $\sigma_{0}$ sur le coefficient de frottement apparent $\operatorname{tg} \varnothing^{*}$ lors des essais de cisaillement sable sur sable. On observe, lorsque $\sigma_{0}$ augmente, une diminution importante de $\operatorname{tg} \varnothing^{\star}$ qui tend vers une valeur asymptotique d'environ 3,5 pour des contraintes normales supérieures à $200 \mathrm{kPa}$.

Ces comportements sont tout à fait analogues à ceux décrits dans les paragraphes $\mathbf{6 . 2}$ et $\mathbf{6 . 3}$ sur l'influence de l'état de surface et de la contrainte normale initiale sur les coefficients de frottement apparent $\mu^{\star}$ déterminés dans des essais d'extraction d'armatures de terre armée. Cette analogie apparaît clairement sur la figure 19 où est également représenté le nuage des valeurs de $\mu^{*}$ mesurées lors de ces essais d'extraction d'armatures de terre armée.

\section{Règles pratiques de dimensionnement}

Les résultats présentés précédemment montrent que le frottement entre un sol frottant et une inclusion dépend de nombreux facteurs, parmi lesquels les plus importants sont la compacité du sol, l'état de surface de l'inclusion et la contrainte normale moyenne s'exerçant initialement sur l'inclusion. On a pu mettre en évidence que ces comportements sont directement liés au phénomène de dilatance dans les sols denses.

Les techniques de renforcement ainsi que les règles pratiques de dimensionnement des inclusions doivent donc tenir compte au maximum de la dilatance susceptible d'être mobilisée dans le sol. Le renforcement sera ainsi particulièrement bien adapté aux sols granulaires denses, ayant une granulométrie bien étalée et continue. Dans les sols peu denses, le procédé d'exécution devra être choisi de façon à créer localement, autour de l'inclusion, une zone de sol compact. Le mode d'exécution a d'ailleurs une

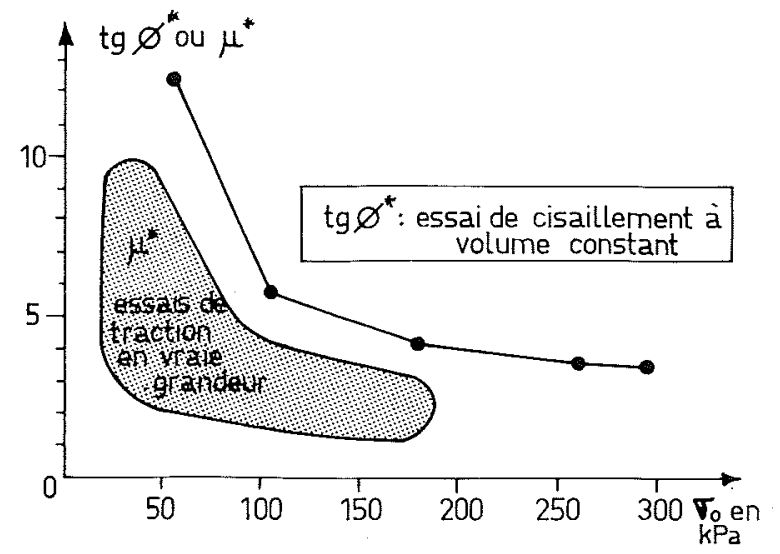

Fig. 19

influence importante sur la mobilisation de la dilatance, et donc sur la valeur du frottement latéral à prendre en compte dans le dimensionnement. On peut distinguer plusieurs modes d'exécution :

1) Mise en place d'inclusions dans un sol rapporté (remblai), pour lequel on maîtrise complètement la nature et la compacité du sol. C'est le cas de la terre armée, où l'on peut encore améliorer le frottement en jouant sur l'état de surface de l'inclusion.

2) Mise en place par battage ou fonçage dans des sols en place. Dans ce cas, le refoulement du sol autour de l'inclusion s'accompagne d'une densification locale qui augmente la valeur de la contrainte normale initiale $\sigma_{0}$. II est difficile de jouer sur l'état de surface de l'inclusion qui, pour faciliter la mise en place, doit rester relativement lisse.

3) Mise en place par forage et scellement par coulis de ciment sans injection. L'inclusion est alors constituée par l'ensemble barre-coulis et son diamètre est celui du forage. Le sol autour de l'inclusion se trouve décomprimé et la contrainte normale initiale est très faible. Ce procédé est a priori à déconseiller, sauf pour des sols présentant une forte compacité naturelle, car dans ce cas la paroi irrégulière du forage constitue un élément favorable qui compense la faible valeur de la contrainte normale initiale $\sigma_{0}$.

4) Mise en place par forage et scellement par injection de coulis sous pression. Comme dans le cas 3 , l'inclusion est constituée par l'ensemble barrecoulis, mais son diamètre peut être sensiblement supérieur à celui du forage par suite de la compressibilité du sol.

L'exécution de micropieux dans des sables lâches avec une pression d'injection voisine de la pression limite du sol a ainsi montré que le diamètre des micropieux pouvait atteindre $25 \mathrm{~cm}$, avec un forage initial de $15 \mathrm{~cm}$. De plus, comme dans le cas 2 , il y a densification du sol et augmentation de la contrainte par refoulement, mais il se crée en plus une surface de contact très irrégulière entre le coulis et le sol, agissant dans le sens d'une meilleure mobilisation de la dilatance.

Les valeurs du frottement que l'on peut proposer en fonction de ces considérations sont les suivantes: 

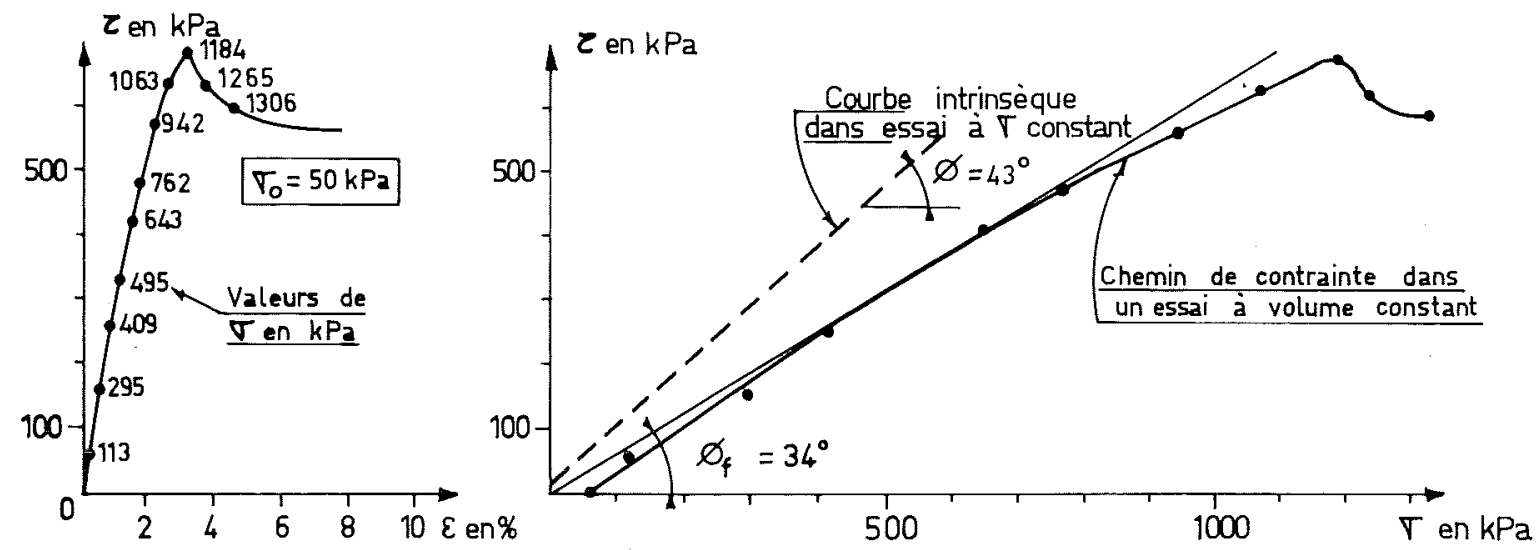

CISAILLEMENT SABLE -SABLE $\quad\left(D_{\Omega}=0,92\right)$

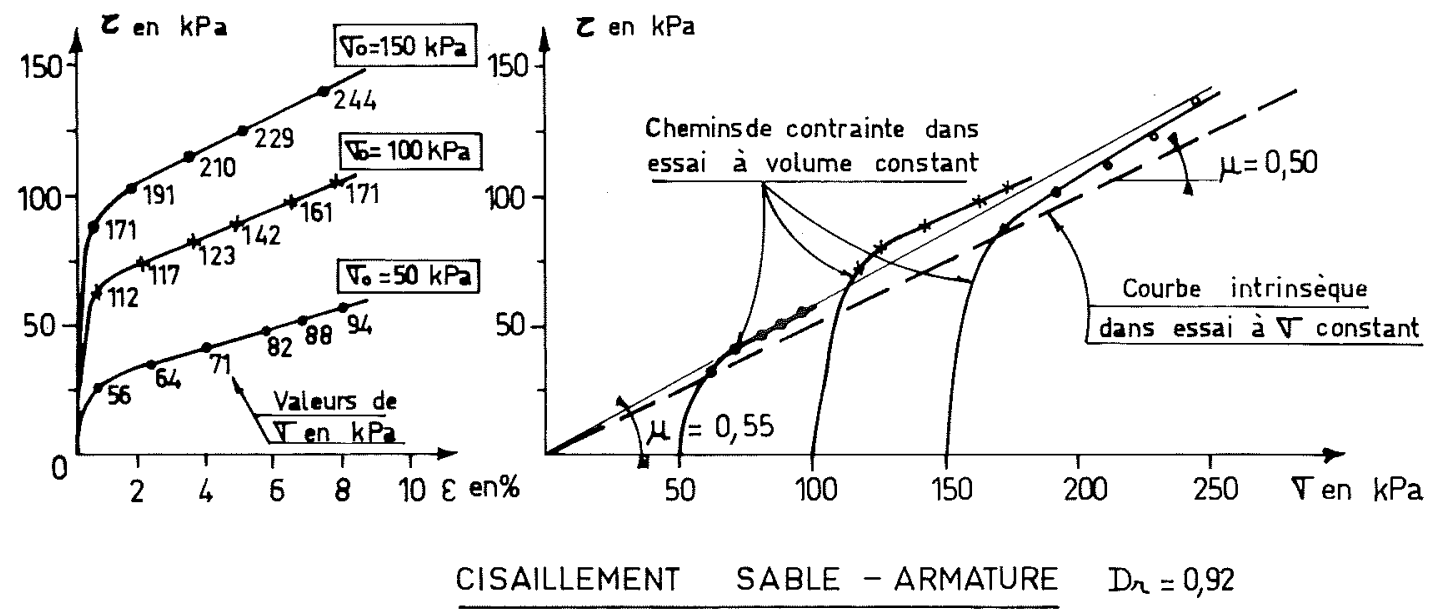

Fig. 17

angle de frottement minéral sur minéral $\varnothing_{\mu}$ de $23^{\circ}$. Cet angle est également très voisin de l'angle de frottement résiduel dans l'essai à contrainte normale constante, lequel est également associé à une variation de volume nulle au cours de la déformation.

Par contre dans le cisaillement à volume constant sable sur plaque métallique, les courbes $(\tau, \varepsilon)$ ne présentent pas de pic, mais un accroissement régulier de la contrainte de cisaillement $\tau$ à partir de très faibles déformations $\varepsilon$. Les chemins de contraintes $(\tau, \sigma)$ montrent que le frottement maximal est mobilisé très rapidement, et qu'il correspond à un coefficient de frottement sol-inclusion $\mu$ égal à tg $29^{\circ}=0,55$, donc assez voisin du coefficient de frottement $\mu=0,50$ mesuré dans un essai de cisaillement sable sur inclusion à contrainte normale constante. Ce comportement s'explique par le fait que dans le cas d'un cisaillement sable sur plaque, l'aspect tridimensionnel et l'effet de la dilatance sont pratiquement négligeables dès que les dimensions de la plaque deviennent importantes.
7.2 Influence de la dilatance et de la contrainte initiale sur le coefficient apparent

Par analogie avec la définition du coefficient de frottement apparent $\mu^{\star}=\frac{\tau_{\max }}{\sigma_{0}}$ dans le cas de la terre armée (cf. $\S$ 4.3), on peut étudier dans le cas d'essais de cisaillement à volume constant, la mobilisation des coefficients de frottement apparent définis par:

$\operatorname{tg} \varnothing^{*}=\frac{\tau}{\sigma_{0}}$ pour le frottement sable sur sable

$$
\mu^{*}=\frac{\tau}{\sigma_{0}} \text { pour le frottement sable sur plaque }
$$

$\tau$ étant la contrainte de cisaillement et $\sigma_{0}$ la contrainte normale en début d'essai.

La figure 18 montre les variations de ces deux coefficients de frottement apparent en fonction de la déformation $\varepsilon$. II apparaît que : 
Terre armée: pour les armatures crénelées, la valeur de $\mu^{*}$ est variable avec la profondeur $z$ en mètres, suivant la loi :

$$
\begin{gathered}
z \leqslant 6 m \quad \mu^{*}=\mu_{0}^{*}-\left(\mu_{0}^{*}-\operatorname{tg} \varnothing\right) \frac{z}{6} \\
z \geqslant 6 m \quad \mu^{*}=\operatorname{tg} \varnothing
\end{gathered}
$$

où $\varnothing$ est l'angle de frottement interne du sol, et $\mu_{0}^{\star}$ une valeur fonction de la granulométrie du sol, définie par son coefficient d'uniformité $\mathrm{C}_{\mathrm{u}}$ :

$$
\mu_{0}^{*}=1,2+\log C_{u}
$$

Pour les armatures lisses, la valeur de $\mu^{*}$ est prise constante et égale au coefficient de frottement réel $\mu$.

Inclusion mise en place par battage ou fonçage : on peut retenir les valeurs de $\tau_{\max }$ déduites des essais pressiométriques pour le calcul du frottement latéral dans le cas des pieux mis en place par refoulement du sol. $\tau_{\max }$ croît de 30 à $100 \mathrm{kPa}$.

Inclusion mise en place par forage et scellement sans pression : dans ce cas, les règles de dimensionnement des pieux mis en place sans refoulement, mais mobilisant spécialement le frottement latéral, sont applicables : $\tau_{\max }$ croît de 50 à $150 \mathrm{kPa}$ lorsque $\mathrm{p}_{\mathrm{f}}$ croît de 30 à $150 \mathrm{kPa}$. Notons que ces valeurs peuvent être très sous-estimées, puisqu'on a pu mesurer des valeurs de $\tau_{\max }$ de $600 \mathrm{kPa}$ pour des barres passives mises en place par scellement sans injection dans des moraines très compactes.

Inclusion mise en place par forage et injection de coulis: on citera les règles DIN 1979 donnant des valeurs minimales de $\tau_{\max }$ de $150 \mathrm{kPa}$ dans les sables et $200 \mathrm{kPa}$ dans les graves, pour des inclusions sollicitées en compression. Lorsque les inclusions sont sollicitées en traction, les règles DIN conseillent de diviser par 2 ces valeurs de $\tau_{\max }$.

Précisons que toutes les valeurs indiquées précédemment constituent des valeurs minimales du frottement latéral unitaire que l'on peut prendre en compte dans le dimensionnement. Notamment dans le cas de renforcement de sols en place, il paraît nécessaire de prévoir des essais d'arrachement d'inclusions mises en place selon le procédé retenu, afin d'apprécier au mieux l'influence de la dilatance, difficile à prendre en compte dans des règles générales.

\section{Remerciements}

Les auteurs remercient M. Frank, Ingénieur au Laboratoire Central des Ponts et Chaussées, des discussions fructueuses qu'ils ont eues avec lui sur certains aspects du frottement des sols granulaires.

\section{Références bibliographiques}

Alimi, Bacot, Lareal, Long, Schlosser (1977). «Etude de l'adhérence sol-armature." Proc. of the 9th Int. Conf. on Soil Mechanics and Found. Eng. Tokyo, 1977.

Alimi (1978). "Critère de choix des matériaux de terre armée. Étude de l'adhérence sol-armature. "Thèse L. C.P.C., Paris, 1978.

Baguelin, Venon (1971). "Influence de la compressibilité des pieux sur la mobilisation des efforts résistants. " Journées du C.F.M.S. : «Le comportement des sols avant la rupture. " Mai 1971.

Bishop (1966). "Shear strength in soils. " 6th Rankine Conference Geotechnique, Vol. 16, n 2, juin 1966.

Cambefort (1964). «Essai sur le comportement en terrain homogène des pieux isolés et des groupes de pieux. " Annales I. T.B.T.P., n 204, décembre 1964.

Dinesh Mohan, Jain, Kumar. «Load Bearing Capacity of Piles. "1963, Géotechnique, Vol. 13, $\mathrm{n}^{\circ} 1$.

Frank (1975). «Étude théorique du comportement des pieux sous charge verticale. Introduction de la dilatance. " Thèse L. C.P. C., Paris, 1975.

Guilloux, Schlosser, Long (1979). "Étude du frottement sable-armature en laboratoire. " C. R. Colloque Int. «Renforcement des sols. » Paris, 1979

Lee, Seed (1967). «Drained strength characteristics of cohesion soils. » A. S. C. E., Journal of Soil Mech. and Found. Eng. SM6, 1967.

Rowe (1962). "The stress-dilatancy relation for static equilibrium of particles in contact. " Proc. of the Royal Society, A269.

Schlosser, Long (1974). «Dimensionnement des ouvrages en terre armée. " École Nationale des Ponts et Chaussées. Paris, 1974.

Schlosser, Elias (1978). «Friction in Reinforced Earth. » A. S. C. E. Convention Pittsburgh, April 24-28, 1928.

Schlosser, Guilloux (1979). "Le frottement solarmature dans les ouvrages en terre armée. »C. R. Coll. Int. «Renforcement des sols. » Paris, mars 1979.

Schlosser, Juran (1980). "Paramètres de calcul des sols artificiellement améliorés. " Proc. of the Eur. Conf. on Soil Mech. and Found. Eng. Brighton, septembre 1980.

Wernick (1978). «Stresses and strains of the surface of anchors. " Revue Française de Géotechnique. $n^{\circ} 3$, janvier 1978. 